\title{
Test of Weak Equivalence Principle with the multi-band timing of the Crab Pulsar
}

\author{
Zhang Yueyang ${ }^{1}$ and Gong Biping ${ }^{1}$ \\ Physics Department, Huazhong University of Science and Technology, Wuhan, China, \\ 430074 \\ zhangyuey@hust.edu.cn \\ bpgong@hust.edu.cn
}

Received __; accepted _ 


\begin{abstract}
Weak Equivalent Principle (WEP) can be tested through the parameterized post-Newtonian parameter $\gamma$, representing the space curvature produced by unit rest mass. The parameter $\gamma$ in turn has been constrained by comparing the arrival times of photons originating in distant transient events, such as gamma-ray bursts, fast radio bursts as well as giant pulses of pulsars. Those measurements normally correspond to an individual burst event with very limited energy bands and signal-to-noise ratio $(\mathrm{S} / \mathrm{N})$. In this letter, the discrepancy in the pulse arrival times of the Crab Pulsar between different energy bands is obtained by the phase difference between corresponding pulse profiles. This allows us to compare the pulse arrival times at the largest energy band differences, between radio and optical, radio and X-ray, radio and gamma-ray respectively. As the pulse profiles are generated by phase-folding thousands of individual pulses, the time discrepancies between two energy bands are actually measured from thousands of events at each energy band, which corresponds to much higher S/N. The upper limit of the $\gamma$ discrepancy set by such an extensively-observed and well-modeled source is as follows: $\gamma_{\text {radio }}-\gamma_{\gamma-\text { ray }}<3.28 \times 10^{-9}$ at the energy difference of $E_{\gamma-\text { ray }} / E_{\text {radio }} \sim 10^{13}, \gamma_{\text {radio }}-\gamma_{X-\text { ray }}<4.01 \times 10^{-9}$ at the energy difference of $E_{X-\text { ray }} / E_{\text {radio }} \sim 10^{9}, \gamma_{\text {radio }}-\gamma_{\text {optical }}<2.63 \times 10^{-9}$ at $E_{\text {optical }} / E_{\text {radio }} \sim 10^{5}$, and $\gamma_{\text {optical }}-\gamma_{\gamma-\text { ray }}<3.03 \times 10^{-10}$ at $E_{\gamma-\text { ray }} / E_{\text {optical }} \sim 10^{8}$. This actually measures the arrival times of freely-falling photons in the gravitational field of the Milky Way with the largest amount of events and with data of the highest $\mathrm{S} / \mathrm{N}$, which tests WEP at the energy band differences that has never been reached before.
\end{abstract}

Subject headings: Gravitation - Pulsars: the Crab Pulsar 


\section{Introduction}

Weak Equivalence Principle (WEP) is an important foundation of general relativity and many other metric theories of gravity. WEP can be tested through the parameterized post-Newtonian (PPN) parameters, such as the parameter $\gamma$, which is defined as how much space curvature is produced by unit rest mass (see Will 2006, 2014). The accuracy of WEP can be obtained by the $\gamma$ discrepancy for particles with different properties, since any gravity theory satisfying WEP predicts the same $\gamma$ value. The time interval required for particles to traverse a given distance is longer in the presence of a gravitational potential $U(\mathbf{r})$ by

$$
\delta t=\frac{1+\gamma}{c^{3}}\left|\int_{\mathbf{r}_{o}}^{\mathbf{r}_{e}} U(\mathbf{r}) d \mathbf{r}\right|,
$$

where $\mathbf{r}_{o}$ and $\mathbf{r}_{e}$ are locations of the observer and the emission site of the particles respectively (Shapiro 1964; Krauss \& Tremaine 1988; Longo 1988). So the $\gamma$ discrepancy can be constrained by the time delay between different particles emitted from an astronomical source.

In previous works, the time delay data used to test WEP are all extragalactic transient sources such as gamma-ray bursts (GRB, e.g. Gao et al. 2015; Sang et al. 2016), fast radio bursts (FRB, e.g. Wei et al. 2015; Tingay \& Kaplan 2016) and TeV blazers (e.g. Wei et al. 2016), with the exception of a 0.4-nanosecond giant pulse of the Crab Pulsar that was used by a recent work of Yang \& Zhang (2016). The time delay between photons of different energies of GRBs and TeV blazers is determined by cross-correlation between light curves obtained from observations at different energy bands (Gao et al. 2015; Wei et al. 2016; Nusser 2016). In other works, the duration time of an abrupt burst event (a FRB, a short GRB or a giant pulse of a pulsar) is used as a representative of the time delay between the highest and lowest energies within the bandpass of the observing telescope (Wei et al. 2015; Sang et al. 2016; Yang \& Zhang 2016; Tingav \& Kaplan 2016; Nusser 2016). The 
gravitational potential in consideration is either a Keplerian potential of the Milky Way for sources not too far away, or a cosmological form of the large-scale structure for sources at redshift $z \gtrsim 0.5$ (Nusser 2016; Tingay \& Kaplan 2016).

In this work, the pulse timing of the Crab Pulsar, a well-studied source with extensive observations at the widest energy coverage, is used to test WEP. And a more realistic gravitational potential of the Milky Way is adopted.

The Crab Pulsar has been extensively observed in energy bands from radio to $\gamma$-ray. The pulse profile of the Crab Pulsar is generated by phase-folding thousands of individual pulses, reaching a very high signal-to-noise ratio $(\mathrm{S} / \mathrm{N})$. It exhibits the feature of two most prominent pulse components which is remarkably similar over almost all the energy bands. The time delays between different bands are very small, which are less than $\sim 300 \mu \mathrm{s}$, corresponding to $\sim 1 \%$ of the spin period (e.g. Kuiper et al. 2003; Oosterbroek et al. 2006). It is thus believed that the similar pulse profile over all energy bands originates in the same emission region. The accurate time delay measurements based on high-S/N data, the well-modeled pulse profile, and the wide energy coverage of the Crab Pulsar's emission makes it a perfect source to test WEP.

Unlike extragalactic sources, the Crab Pulsar locates in the Milky Way and is very close to the Galactic disc. The gravitational potential of the Milky Way can not be simply considered as a Keplerian potential, but a more complex form.

A brief description of the method of testing WEP is presented in Section 2. Time delay measurements of the Crab Pulsar in literature are discussed in Section 3. In Section 4, the constraints of the $\gamma$ discrepancy using the timing of the Crab Pulsar are shown and compared with previous works. A summary is given in Section 5 . 


\section{Method description}

In principle, the observed time delay between photons of two different energy bands consists of five terms (Gao et al. 2015; Wei et al. 2015, 2016):

$$
\Delta t_{\text {obs }}=\Delta t_{i n t}+\Delta t_{L I V}+\Delta t_{\text {spe }}+\Delta t_{D M}+\Delta t_{\text {gra }}
$$

In Eq. 2, $\Delta t_{\text {int }}$ is the intrinsic time delay between two photons, which is determined by the source's radiation mechanism and other characteristics that affect the time delay of photons with different energies. The term $\Delta t_{L I V}$ is the time delay due to the effect of Lorentz invariance violation via an energy-dependent velocity of light. And $\Delta t_{\text {spe }}$ is the time delay caused by special-relativistic effects for photons with non-zero rest masses.

The time delay $\Delta t_{D M}$ stems from the dispersion of the line-of-sight free electrons. It is larger for a photon with lower energy and vanishes as the photon energy becomes infinite. In the standard data reduction process of pulsar timing like TEMPO2 (Hobbs et al. 2006), the times of arrival of a pulsar are converted to the "infinite" frequency (which is the so called de-dispersion procedure). Therefore, $\Delta t_{D M}$ is negligible in this work.

From Eq. 1, the time delay $\Delta t_{\text {gra }}$ represents the difference in arrival times between two different photons originated in the gravitational potential $U(\mathbf{r})$, as

$$
\Delta t_{\text {gra }}=\frac{\gamma_{1}-\gamma_{2}}{c^{3}}\left|\int_{\mathbf{r}_{o}}^{\mathbf{r}_{e}} U(\mathbf{r}) d \mathbf{r}\right|,
$$

where $\gamma_{1}$ and $\gamma_{2}$ are the $\gamma$ values of two photons with different energies.

The timing of the Crab Pulsar shows that the low energy photons arrive later than the high energy photons $\left(\Delta t_{o b s}>0\right)$, which is believed to be mainly due to the intrinsic time delay, so that the relationship of $\Delta t_{i n t}>0$ also holds. Assuming $\Delta t_{\text {gra }}>0$, we have $\gamma_{1}-\gamma_{2}>0$ by Eq. 3. If we also assume $\Delta t_{L I V}+\Delta t_{\text {spe }}>0$, so 
$\Delta t_{\text {gra }}=\Delta t_{\text {obs }}-\Delta t_{\text {int }}-\left(\Delta t_{L I V}+\Delta t_{\text {spe }}\right)<\Delta t_{\text {obs }}$. And hence,

$$
\Delta t_{o b s}>\frac{\gamma_{1}-\gamma_{2}}{c^{3}}\left|\int_{\mathbf{r}_{o}}^{\mathbf{r}_{e}} U(\mathbf{r}) d \mathbf{r}\right|
$$

This gives the most conservative constraint of $\gamma_{1}-\gamma_{2}$.

We adopt a more realistic gravitational potential form, considering it as a twocomponent system, including a Miyamoto-Nagai disc (Miyamoto \& Nagai 1975),

$$
\Phi_{\text {disc }}(R, z)=-\frac{G M_{d}}{\sqrt{R^{2}+\left(r_{a}+\sqrt{Z^{2}+r_{b}^{2}}\right)^{2}}},
$$

and a NFW (Navarro, Frenk \& White 1996) dark matter halo,

$$
\Phi_{\text {halo }}(r)=-\frac{G M_{v i r}}{r[\log (1+c)-c /(1+c)]} \log \left(1+\frac{r}{r_{v i r}}\right)
$$

with parameters adopted from (Gómez et al. 2010). In Eq. 5, $R$ and $z$ are the radial distance and the height in the cylindrical coordinate system, and $r$ in Eq. 6 is the radial distance in the spherical coordinate system, which satisfies $r=\sqrt{R^{2}+z^{2}}$. Table 1 lists the values of parameters used in this work.

So, the gravitational potential is the sum of the two potentials described by Eq. 5 and Eq. 6, as $U(R, z)=\Phi_{\text {disc }}(R, z)+\Phi_{\text {halo }}(r)$.

Integrating along a straight line from the Sun to the Crab Pulsar, $U(R, z)$ can be characterized as $U(R)$, since $z$ is a function of $R$. Then we have

$$
\gamma_{1}-\gamma_{2}<\frac{\Delta t_{o b s} c^{3}}{\left|\int_{R_{o}}^{R_{e}} U(R) d R\right|},
$$

where $R_{o}$ stands for the radial distance of the Sun relative to the Galactic center and $R_{e}$ is the radial distance of the Crab Pulsar. We adopt $R_{o}=8.3 \mathrm{kpc}$ and $z_{o}=15 \mathrm{pc}$. The distance from the Crab Pulsar to the Sun is $d=2.0 \mathrm{kpc}$ (Kaplan et al. 2008). The Galactic coordinate of the Crab Pulsar is $\left(l \approx 184.56^{\circ}, b \approx-5.78^{\circ}\right) . R_{e}$ and $z_{e}$ can be 
easily calculated by $R_{e}=\sqrt{R_{o}^{2}+(d \cos b)^{2}-2 R_{o} d \cos b \cos \left(360^{\circ}-l\right)} \approx 10.3 \mathrm{kpc}$ and $z_{e}=z_{o}+d \sin b \approx-186 \mathrm{pc}$. The relation between $z$ and $R$ is $z=-0.1005 R+0.8492$.

Then, the discrepancy between $\gamma_{1}$ and $\gamma_{2}$ corresponding to two photons with different energies relates with their time discrepancy $\Delta t_{\text {obs }}$, by,

$$
\gamma_{1}-\gamma_{2}<1.167 \times 10^{-5} \mathrm{~s}^{-1} \Delta t_{o b s}
$$

Consequently, WEP can be tested by Eq. 8 with the values of $\Delta t_{\text {obs }}$ between various energy bands.

\section{Time delay measurements of the Crab Pulsar}

The latest time delay measurements of the Crab Pulsar are summarized in Table 2, for optical, X-ray and $\gamma$-ray pulses in comparison with radio pulses. We also obtain a optical- $\gamma$-ray time delay by comparing data of optical and $\gamma$-ray in this table. Most of the measurements have used the Crab Pulsar Monthly Ephemeris of the Jodrell Bank

Observatory (Lvne et al. 1993) to compare with the pulse arrival times of higher energy bands, except Oosterbroek et al. (2008) and Abdo et al. (2010) who carried out their own radio observations.

Unlike GRBs and TeV blazers, the time delays of which are determined by crosscorrelation between light curves at different energy bands, the arrival times of a pulsar can be directly measured because of the prominent peak displayed in the pulse profile. The pulse arrival time of the Crab Pulsar at each energy band is defined by the phase of the main pulse peak. And the time delay between two energy bands is calculated from the phase discrepancy between them.

The highly stable profile of a pulsars allows one to phase-fold thousands of individual 
pulses, so that the integrated pulse profile obtained is of a very high $\mathrm{S} / \mathrm{N}$. This is analogous to adding up thousands of identical transient events to obtain the overall light curve. Taking Abdo et al. (2010) for example, the Crab Pulsar was observed in radio band by Nançay radio telescope with an integration time of 1 minute, which means the integrated pulse profile is generated by phase-folding $\sim 1800$ periods, resulting in an $\mathrm{S} / \mathrm{N}$ of $\gtrsim 1000$ (see Figure 1 of Abdo et al. 2010). The $\gamma$-ray emission of the Crab Pulsar is recorded as isolated photon events on the pulsar's coordinates in a time sequence. $14,563 \pm 240$ pulsed $\gamma$-ray photons were obtained from 248 days of data. Based on the radio ephemeris, the times of photon events are converted into phases within the pulsar period. In other words, the pulse profile of $\gamma$-ray emission is generated by phase-folding data of 248 days. The $\mathrm{S} / \mathrm{N}$ of the $\gamma$-ray profile is still $\gtrsim 100$ in spite of much lower $\gamma$-ray flux intensity.

The uncertainty of the time delay measurements is usually separated into two parts, the statistical and systematic uncertainty respectively. The former comes from the procedure of fitting the pulse profiles of the two bands in comparison by a template of the profile, which aims to accurately determine the peak of the pulse profile. The latter one stems from the error of clocks used by the instruments and the error of the dispersion measure obtained from the radio observation. Here the two terms are added in quadrature.

We selected the radio-optical and radio- $\gamma$-ray time delays with the smallest uncertainties in the test, i.e. $\Delta t_{\text {radio-optical }}=255 \pm 21 \mu \mathrm{s}$ and $\Delta t_{\text {radio- }- \text {-ray }}=281 \pm 24 \mu \mathrm{s}$. The measurement of Rots et al. (2004) $\Delta t_{\text {radio-X-ray }}=344 \pm 40 \mu$ s deviates from the rest of radio-X-ray data considerably. In order to make a conservative calculation, it is still selected because it corresponds to the largest discrepancy between the radio and X-ray data. The optical- $\gamma$-ray time delay $\Delta t_{\text {optical- }- \text {-ray }}=26 \pm 32 \mu \mathrm{s}$ is also included in the test. 


\section{Result and discussion}

Consequently, WEP can be tested via Equation 8 by the discrepancies of $\gamma$ values between different energy bands:

$$
\begin{gathered}
\gamma_{\text {radio }}-\gamma_{\text {optical }}<2.63 \times 10^{-9}, \\
\gamma_{\text {radio }}-\gamma_{X-\text { ray }}<4.01 \times 10^{-9}, \\
\gamma_{\text {radio }}-\gamma_{\gamma-\text { ray }}<3.28 \times 10^{-9}, \\
\gamma_{\text {optical }}-\gamma_{\gamma-\text { ray }}<3.03 \times 10^{-10},
\end{gathered}
$$

As a comparison, the new constraints of the $\gamma$ discrepancy (Eq. 9,12) and some of the best previous results are listed in Table 3. The parameter $E_{\text {high }} / E_{l o w}$ is defined to indicate the energy band difference in each comparison, where $E_{\text {high }}$ and $E_{\text {low }}$ are the higher and lower energy bands respectively.

The first three constraints Eq. 9.11 test WEP by comparing radio pulses with those of higher energy bands. WEP has never been tested with these energy band differences before. Moreover, the radio-X-ray and radio- $\gamma$-ray constraints have the largest energy band differences of $10^{9}$ and $10^{13}$ respectively, with 3 to 7 orders larger than those of previous works.

In previous works, the test with the largest energy band difference and the relatively most stringent constraint is from Nusser (2016), which gives $\gamma_{e V}-\gamma_{M e V}<2.3 \times 10^{-10}(3 \sigma)$, or $\gamma_{e V}-\gamma_{M e V}<1.3 \times 10^{-11}(2 \sigma)$. In contrast, Eq. 12 sets a similarly stringent constraint

at the similar energy range, but with an energy band difference 2 orders larger than Nusser $(2016)$.

In general, the new results extend the test of WEP to the largest energy band differences. And in the case of comparable constraints of the $\gamma$ discrepancy at the 
optical- $\gamma$-ray comparison, the energy band difference of the new result is 2 orders larger than Nusser (2016).

Up to date, the most stringent constraint of the $\gamma$ discrepancy is $\gamma(8.15 \mathrm{GHz})-$ $\gamma(10.35 \mathrm{GHz})<(0.6-1.8) \times 10^{-15}$, obtained from a 0.4 -nanosecond giant pulse duration time of the Crab Pulsar by Yang \& Zhang (2016), but it only covers a very narrow radio frequency band of $2.2 \mathrm{GHz}$.

\section{Summary}

The accuracy of WEP can be characterized by the discrepancy in the parameter $\gamma$, for photons with different energies. Unlike the transient signals such as GRBs and giant pulses of pulsars, a non-transient signal, the timing of the Crab Pulsar with high-S/N data, is applied in the test of WEP at multiple energy bands from radio to $\gamma$-ray. The test of WEP is thus extended to energy band differences up to $E_{\text {high }} / E_{\text {low }} \sim 10^{13}$ which has never been achieved before. The new constraint for the optical- $\gamma$-ray test is comparable to the best previous result but with a 2-order larger energy band difference.

Comparing with previous works, the new method has three advantages:

- A more realistic gravitational potential of the Milky Way is applied when calculating the time delay $\Delta t_{g r a}$, which makes the constraint more accurate.

- The pulsation of the Crab Pulsar is stable, periodic, and always available for observation. The high-S/N pulse profile obtained by the phase-folding technique makes the time delay data of the pulsar can be measured with high precisions.

- If WEP was not satisfied by a gravity theory, the parameter $\gamma$ would be different for photons with different energies. Then the photons with the most different energies 
would be most likely to reveal the $\gamma$ discrepancy. The multi-band pulsation of the Crab Pulsar from radio to $\gamma$-ray provides a great opportunity to test WEP at the largest energy band difference.

The intrinsic time delay of the Crab Pulsar is most likely due to the different locations of the emission sites of different energy bands, as the pulse periods of different energy bands are identical. Under such a circumstance, a time delay of $\sim 300 \mu$ s can be explained by two reasons: firstly the emission region of radio band locates at an emission region $90 \mathrm{~km}$ lower than that of the higher band, and secondly between the radio beam and that of the higher band exists an angle of $\sim 3.3^{\circ}$. The detailed structure and geometry of the emission region of the Crab Pulsar will allow us to get more precise values of $\Delta t_{i n t}$. With more understanding of the emission region of the Crab Pulsar, more stringent limits on WEP are expected in the future.

We wish to thank Dick Manchester and George Hobbs for helpful discussion and suggestions. And we thank Hao Tong and Mingyu Ge for communication on the latest progress on the time delay measurements of the Crab Pulsar. This research is supported by the National Natural Science Foundation of China, under the grant NSFC11373018 and NSFC11503006. We also thank for the support of the grant Beyond the Horizons 2012. 
Table 1. Parameters of the gravitational potential used in Eq. 5] and Eq. 6. cited from Gómez et al. (2010).

\begin{tabular}{cl}
\hline \hline \multicolumn{1}{c}{ Disc } & \multicolumn{1}{c}{ Halo } \\
\hline & \\
$M_{d}=7.5 \times 10^{10} \mathrm{M}_{\odot}$ & $M_{\text {vir }}=9 \times 10^{11} \mathrm{M}_{\odot}$ \\
$r_{a}=5.4 \mathrm{kpc}$ & $r_{v i r}=250 \mathrm{kpc}$ \\
$r_{b}=0.3 \mathrm{kpc}$ & $c=13.1$ \\
\hline
\end{tabular}


Table 2. The time delays of the Crab Pulsar for optical, X-ray and $\gamma$-ray pulses in comparison with radio pulses.

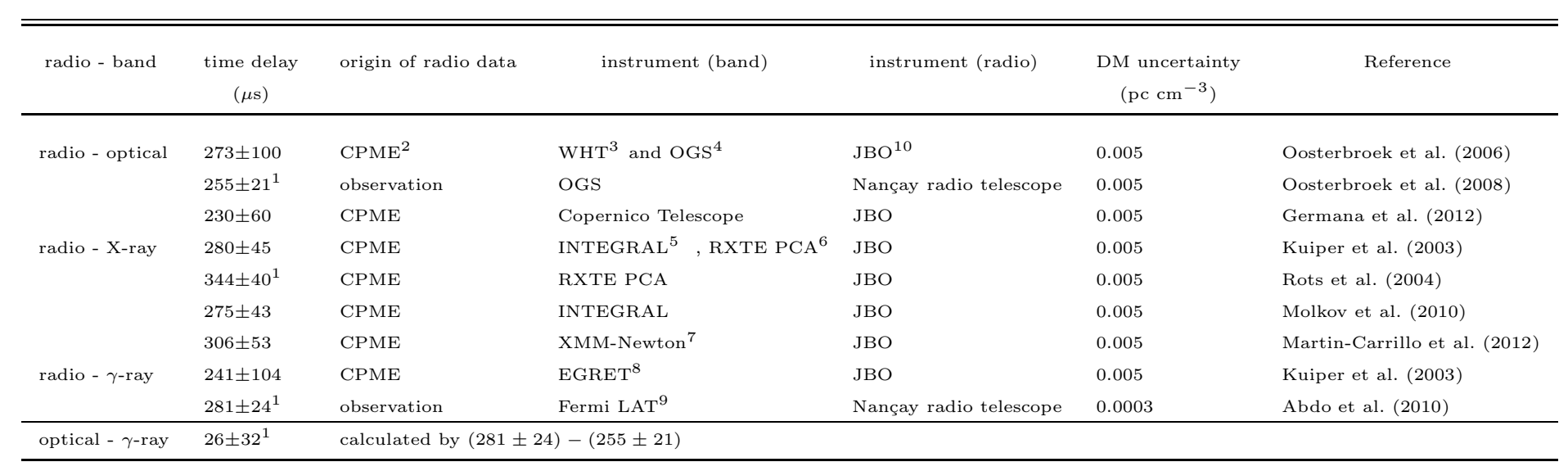

${ }^{1}$ The data marked with "1" are used in the test of this letter.

${ }^{2}$ Crab Pulsar Monthly Ephemeris

${ }^{3}$ William Hershel Telescope

${ }^{4}$ Optical Ground Station Telescope

${ }^{5}$ International Gamma-Ray Astrophysics Laboratory

${ }^{6}$ Proportional Counter Array on the Rossi X-ray Timing Explorer

${ }^{7}$ X-ray Multi-Mirror Mission

${ }^{8}$ Energetic Gamma Ray Experiment Telescope

${ }^{9}$ Fermi Large Area Telescope

${ }^{10}$ Jodrell Bank Observatory 
Table 3: The upper limits of the $\gamma$ discrepancy given by this work and previous works

\begin{tabular}{lllll}
\hline \hline Source name & Test particles and energy bands & Upper limit of $\Delta \gamma$ & $E_{\text {high }} / E_{\text {low }}$ & Reference \\
\hline Crab Pulsar & photon(radio) - photon(optical) & $2.63 \times 10^{-9}$ & $\sim 10^{5}$ & Eq. 9 \\
Crab Pulsar & photon(radio) - photon(X-ray) & $4.01 \times 10^{-9}$ & $\sim 10^{9}$ & Eq. [10 \\
Crab Pulsar & photon(radio) - photon $(\gamma$-ray) & $3.28 \times 10^{-9}$ & $\sim 10^{13}$ & Eq. [1] \\
Crab Pulsar & photon(optical) - photon $(\gamma$-ray) & $3.03 \times 10^{-10}$ & $\sim 10^{8}$ & Eq. 12 \\
\hline GRB 080319B & photon $(e V)$ - photon $(\mathrm{MeV})$ & $2.3 \times 10^{-10}(3 \sigma)$ & $10^{6}$ & Nusser $(2016)$ \\
& & $1.3 \times 10^{-11}(2 \sigma)$ & & \\
Crab Pulsar (giant pulse) & photon $(8.15 \mathrm{GHz})$ - photon $(10.35 \mathrm{GHz})$ & $(0.6-1.8) \times 10^{-15}$ & $\sim 1.2$ & Yang \& Zhang (2016) \\
\hline
\end{tabular}




\section{REFERENCES}

Abdo, A., Axelsson, M., Baldini, L. et al. 2010, ApJ, 708, 1254

Amsler, C., Doser, M., Antonelli, M. et al. 2008, PhLB, 667, 1

Gao, H., Wu, X., Mészáros, P. 2015, ApJ, 810, 121

Germanà, C., Zampieri, L., Barbieri, C. et al. 2012, A\&A, 548, A47

Gómez, F., Helmi, A., Brown, A. et al. 2010, MNRAS, 408, 935

Hobbs, G., Edwards, R. \& Manchester, R. 2006, MNRAS, 369, 655

Kaplan, D., Chatterjee, S., Gaensler, B. et al. 2008, ApJ, 677, 1201

Krauss, L. M. \& Tremaine, S. 1988, Phys. Rev. Lett., 60, 176

Kuiper, L., Hermsen, W., Walter, R. et al. 2003, A\&A, 411, L31

Longo, M. J. 1998, Phys. Rev. Lett., 60, 173

Lyne, A., Pritchard, R. \& Graham Smith, F. 1993, MNRAS, 265, 1003

Martin-Carrillo, A., Kirsch, M., Caballero, I. et al. 2012, A\&A, 545, A126

Miyamoto, M. \& Nagai, R. 1975, PASJ, 27, 533

Molkov, S., Jourdain, E., Roques, J. 2010, ApJ, 708, 403

Navarro, J. F., Frenk, C. S. \& White, S. D. M. 1996, ApJ, 462, 563

Nusser, A. 2016, ApJ, 821, L2

Oosterbroek, T., Cognard, I., Golden, A. et al. 2008, A\&A, 488, 271

Oosterbroek, T., de Bruijne, J., Martin, D. et al. 2006, A\&A, 456, 283 
Rots, A., Jahoda, K., Lyne, A. 2004, ApJ, 605, L129

Sang, Y., Lin, H.-N., \& Chang, Z. 2016, MNRAS, 460, 2282

Shapiro, I. I. 1964, Phys. Rev. Lett., 13, 789

Tingay, S. J. \& Kaplan, D. L. 2016, ApJ, 820, L31

Wei, J., Hao, H., Wu, X. et al. 2015, Phys. Rev. Lett., 115, 261101

Wei, J., Wang, J., Gao, H. et al. 2016, ApJ, 818, L121

Will, C. M. 2006, LRR, 9, 3

Will, C. M. 2014, LRR, 17, 4

Yang, Y.-P. \& Zhang, B. 2016, Phys. Rev. D, 94, 101501 